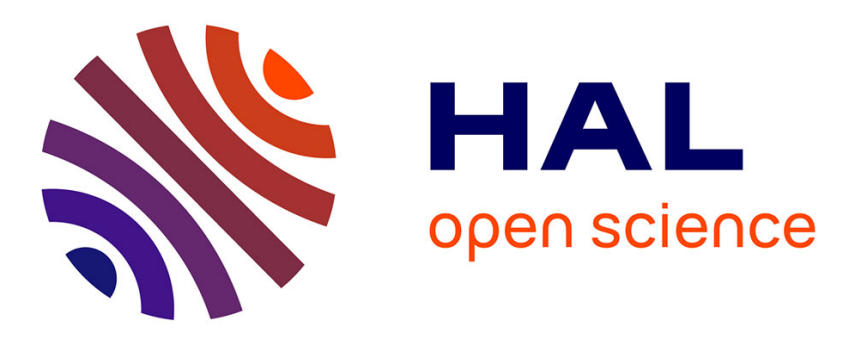

\title{
Revised Theoretical Model on Enantiocontrol in Phosphoric Acid Catalyzed H -Transfer Hydrogenation of Quinoline
}

Julien Pastor, Elixabete Rezabal, Arnaud Voituriez, Jean-François Betzer, Angela Marinetti, Gilles Frison

\section{To cite this version:}

Julien Pastor, Elixabete Rezabal, Arnaud Voituriez, Jean-François Betzer, Angela Marinetti, et al.. Revised Theoretical Model on Enantiocontrol in Phosphoric Acid Catalyzed H Transfer Hydrogenation of Quinoline. Journal of Organic Chemistry, 2018, 83 (5), pp.2779-2787. 10.1021/acs.joc.7b03248 . hal-01927648

\section{HAL Id: hal-01927648 \\ https://hal.science/hal-01927648}

Submitted on 11 Jun 2019

HAL is a multi-disciplinary open access archive for the deposit and dissemination of scientific research documents, whether they are published or not. The documents may come from teaching and research institutions in France or abroad, or from public or private research centers.
L'archive ouverte pluridisciplinaire HAL, est destinée au dépôt et à la diffusion de documents scientifiques de niveau recherche, publiés ou non, émanant des établissements d'enseignement et de recherche français ou étrangers, des laboratoires publics ou privés. 


\title{
Revised theoretical model on enantiocontrol in phosphoric acid catalyzed $\boldsymbol{H}$ - transfer hydrogenation of quinoline
}

Julien Pastor ${ }^{\mathrm{a}}$, Elixabete Rezabal ${ }^{\mathrm{a}, \mathrm{b}}$, Arnaud Voituriez $^{\mathrm{c}}$, Jean-François Betzer ${ }^{\mathrm{c} *}$, Angela Marinetti $^{\mathrm{c}}$, Gilles Frison ${ }^{\mathrm{a} *}$

${ }^{a}$ LCM, CNRS, Ecole polytechnique, Université Paris-Saclay, 91128 Palaiseau, France.

${ }^{\mathrm{b}}$ Kimika Fakultatea, Euskal Herriko Unibertsitatea UPV/EHU, Donostia International Physics Center (DIPC), P.K. 1072, 20080 Donostia, Euskadi, Spain

${ }^{\mathrm{c}}$ Institut de Chimie des Substances Naturelles, CNRS UPR 2301, Université Paris-Sud, Université Paris-Saclay, 1 Avenue de la Terrasse, 91198 Gif-sur-Yvette, France.

* e-mail : gilles.frison@polytechnique.edu ; jean-francois.betzer@cnrs.fr

\begin{abstract}
The enantioselective $H$-transfer hydrogenation of quinoline by Hantzsch ester is a relevant example of Brønsted acid catalyzed cascade reactions, with phosphoric acid being privileged catalysts. The generally accepted mechanism points out the hydride transfer step as the rate- and stereo-determining step, however computations based on these models do not totally fit with experimental observations. We hereby present a computational study that enlightens the stereochemical outcome and quantitatively reproduces the experimental enantiomeric excesses in a series of $H$-transfer hydrogenations. Our calculations suggest that the high stereocontrol usually attained with BINOL-derived phosphoric acids results mostly from the steric constraints generated by an aryl substituent of the catalyst, which hinders the access of the Hantzsch ester to the catalytic site and enforces approach through a specific way. It relies on a new model involving the preferential assembly of one of the stereomeric complexes formed by the chiral phosphoric acid and the two reaction partners. The stereodetermining step thus occurs prior to the $H$-transfer step.
\end{abstract}


Homogeneous catalysis is recognized as one of the most important tools toward sustainable chemical processes. ${ }^{1}$ Therefore, the past decade has witnessed the continuous development of synthetic protocols based on organocatalysis, ${ }^{2,3}$ organometallic catalysis, ${ }^{4-6}$ as well as on multicatalysis. ${ }^{7,8}$ Most of these developments rely exclusively on experimental approaches. However, due to their basically experimental nature, both the development of new catalytic protocols and optimization of the established ones are expensive and time-consuming. Challenges in optimizing catalytic processes result principally from the broad lack of knowledge on reaction mechanisms and intermediates involved in the chemical transformations on the molecular level. Computational approaches are suited to enlighten mechanistic and selectivity issues, as they provide detailed information on intermediates and transition-state structures along potential energy surfaces. ${ }^{9-15}$ They have emerged as interesting tools, often complementary to experiments, in the design and development of catalysts with improved activity and selectivity, in both enzymatic, ${ }^{16-18}$ heterogeneous, ${ }^{19}$ and homogeneous catalysis. ${ }^{20-23}$

Despite their widespread success, ${ }^{24-26}$ computational methods often show their limits when it comes to give rational to, and/or to predict enantioselectivity levels. Strategies developed so far to predict enantiomeric excess (ee) in catalytic reactions include the reverse-docking approach ${ }^{27}$ and quantitative structure-selectivity relationships analyses, which rely on computed physicochemical properties of the catalyst, ${ }^{28-34}$ as well as screening methods based on experimental ${ }^{1} \mathrm{H}$ NMR data. ${ }^{35}$ Moreover, modeling of diastereomeric transition states ${ }^{36-38}$ have been reported for catalytic reactions such as rhodium-catalyzed hydrogenations, ${ }^{39}$ prolinecatalyzed intermolecular aldol reactions ${ }^{40}$ hydrophosphonylation of imines ${ }^{41}$ and pyridine $\mathrm{N}$ oxide-catalyzed allylation and propargylation reactions. ${ }^{42,43}$ Nevertheless, even when a detailed knowledge of the reaction mechanism is available, delivering of predictions consistent with the experimental results in terms of enantioselectivity remains highly challenging. This is mainly because small energy differences between the transition states leading to enantiomeric products suffice to significantly change the enantiomeric excesses. Moreover, the large size and flexibility of the molecular systems usually involved exacerbate even more the challenges of calculation tasks. $^{44-46}$

In the field of organocatalysis, chiral Phosphoric Acids (PA) play a unique role and their use has experienced impressive increase ${ }^{47,48}$ since the seminal work of Akiyama ${ }^{49}$ and Terada $^{50}$ in 2004. The scarce development of theoretical approaches allowing rationalization and prediction of the 
stereoselectivity in PA organocatalysis and, most particularly, in one of its benchmark reactions, the $H$-transfer hydrogenation of quinoline by Hantzsch esters (Scheme 1 ), ${ }^{51}$ motivated us to start studies in this field. Our initial goal was to evaluate the ability of DFT calculations to reproduce the experimentally observed stereoselectivity. Ultimately, this work led us to re-examine the origin of the enantioselectivity in these reactions.

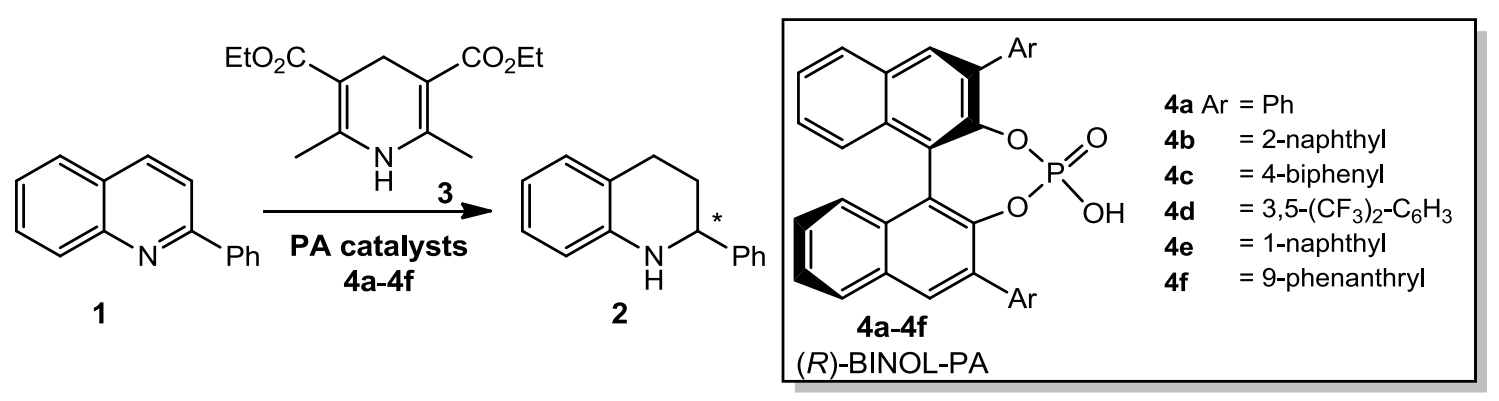

Scheme 1. $H$-transfer hydrogenation of 2-phenylquinoline with Hantzsch esters catalyzed by $(R)$ BINOL-derived phosphoric acids.

Leading examples of enantioselective organocatalytic $H$-transfer hydrogenations of quinolines have been reported by Rueping et al. by using the BINOL-derived phosphoric acids $(\boldsymbol{R})-\mathbf{4}$ as the catalysts (Scheme 1). ${ }^{52}$ The 1,2,3,4-tetrahydroquinoline $(\boldsymbol{S})$-2 was mainly obtained with ee going from $5 \%$ to $97 \%$ by using PA with different aryl substituents on their 3,3'-positions (see Table 1). Such a wide range of enantioselectivity levels poses an interesting challenge for computational modeling that should be able to reflect and give a rational for the observed variations.

Modeling of these enantioselective processes requires, first of all, a thorough knowledge of the reaction mechanism and, then, a proper description of the spatial arrangement of intermediates and transition states in the stereodetermining step. Previous studies on $H$-transfer hydrogenations catalyzed by PA have suggested a reaction mechanism made of two subsequent reduction cycles, each of them involving one equivalent of Hantzsch ester (HE) $3,{ }^{52}$ and also that PA acts as an acid-base bifunctional catalyst with simultaneous activation of the two reaction partners ${ }^{53,54}$ (Scheme 2). The first reduction cycle (not shown) leads to the dihydroquinoline (DHQ) 5, which then enters in the second reduction cycle which is generally assumed to involve $(i)$ formation of the two-component iminium phosphate complex, 6, from the DHQ and the PA catalyst 4; (ii) 
formation of a three-component complex, 7, by coordination of HE 3 to $\mathbf{6}$; (iii) hydride transfer from HE to DHQ; and (iv) dissociation of the final tetrahydroquinoline $\mathbf{2}$ from the catalyst.

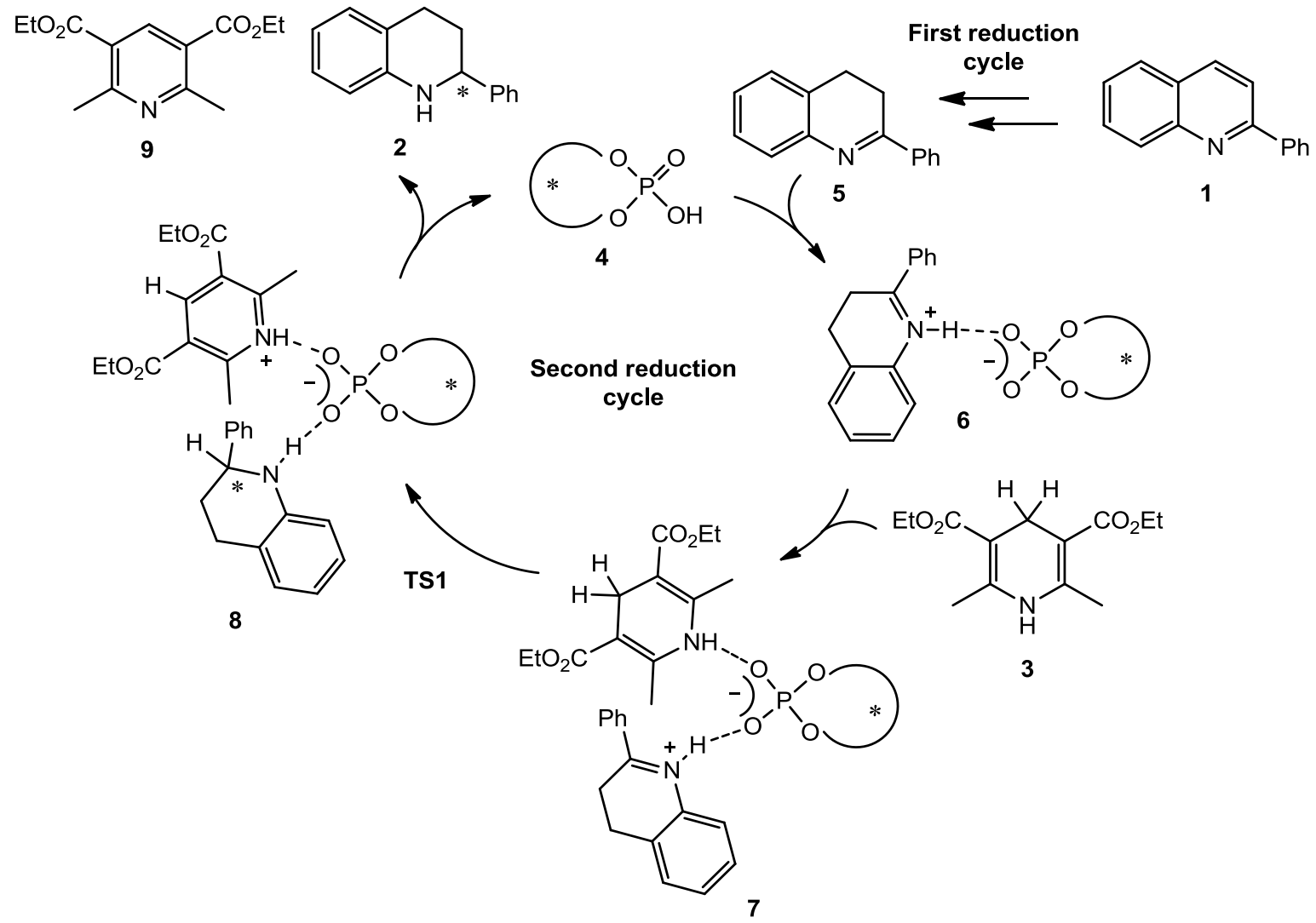

Scheme 2. Proposed catalytic cycle for the $H$-transfer hydrogenation of 2-phenylquinoline with Hantzsch esters catalyzed by phosphoric acids. When necessary, in the following, the name of the molecules will include the $(R)$-, pro- $(R)-,(S)$ - or pro- $(S)$ - prefix to indicate the stereochemistry of the reduced product 2 and its intermediate precursors, and will be completed by the a-h letter to precise the nature of the catalyst.

The hydride transfer step $(\mathbf{7} \rightarrow \mathbf{8}$ ), which produces the stereogenic center, has been previously investigated by Himo et al. ${ }^{54}$ by applying the DFT approach to a simplified PA catalyst, i.e. a biphenol-derived PA with mesityl substituents on its 3,3'-positions. This study allowed identification of two transition states, with the one leading to the major enantiomer of the product being located $15 \mathrm{~kJ} / \mathrm{mol}$ below the other. 


\section{Results and discussion}

These prominent results were taken as starting point for our mechanistic studies. We have analyzed the hydride transfer step through optimization of the geometry of intermediates $\mathbf{7}$ and $\mathbf{8}$ and the corresponding transition state TS1, for pathways leading to the $(S)$ and $(R)$ enantiomers respectively. Calculations have been performed on the six $(R)$-BINOL-derived phosphoric acid 4a-f used experimentally by Rueping et al., ${ }^{52}$ as well as on the mesityl substituted (4g) and the unsubstituted PA 4h (Table 1). All calculations were carried out at the DFT IEFPCM(benzene)M06/6-311++G(2d,2p)//M06/6-31G(d,p) level of theory (see the Supporting Information for details) considering the whole system. The only simplification with respect to the real system consisted in replacing the ethyl moieties of the HE 3 by methyl groups, as already done previously. ${ }^{34,53}$

Table 1. Experimental and calculated enantiomeric excesses, based on the initial hypothesis indicating step $\mathbf{7} \rightarrow \mathbf{8}$ as the enantiodetermining step, for the $H$-transfer hydrogenation of quinolines catalyzed by $(R)$-BINOL-derived PA.

\begin{tabular}{|c|c|c|c|c|c|c|}
\hline \multirow[b]{2}{*}{ entry } & \multirow[b]{2}{*}{ Cat. } & \multirow[b]{2}{*}{$\mathrm{Ar}$} & \multirow{2}{*}{$\begin{array}{l}\text { Exper. }^{\mathrm{a}} \\
\text { ee }[\%]^{\mathrm{a}}\end{array}$} & \multicolumn{3}{|c|}{ DFT calculations } \\
\hline & & & & $\Delta \Delta \mathrm{G}^{\mathrm{TS} 1 \mathrm{~b}}$ & $e e[\%]^{\mathrm{c}}$ & $\begin{array}{c}\text { Major } \\
\text { enantiomer }\end{array}$ \\
\hline 1 & $4 a$ & $\mathrm{Ph}$ & 5 & 11.2 & 98 & $\mathrm{R}$ \\
\hline 2 & $4 b$ & 2-naphtyl & 26 & 7.9 & 92 & $\mathrm{R}$ \\
\hline 3 & $4 c$ & 4-biphenyl & 35 & -2.5 & 47 & S \\
\hline 4 & $4 d$ & $3,5-\left(\mathrm{CF}_{3}\right)_{2}-\mathrm{C}_{6} \mathrm{H}_{3}$ & 72 & -14.0 & 99 & S \\
\hline 5 & $4 e$ & 1-naphtyl & 84 & -6.1 & 84 & $\mathrm{~S}$ \\
\hline 6 & $4 f$ & 9-phenanthryl & 97 & -20.4 & 100 & S \\
\hline 7 & $4 g$ & mesityl & - & $-15.8^{\mathrm{d}}$ & 100 & $S$ \\
\hline 8 & $4 h$ & $\mathrm{H}$ & - & -3.2 & 57 & $\mathrm{~S}$ \\
\hline \multicolumn{7}{|c|}{ 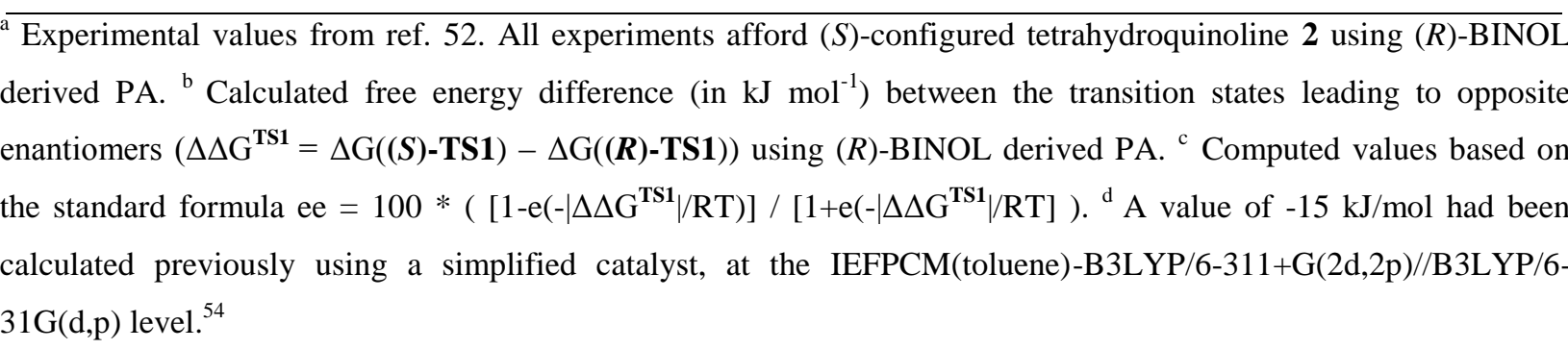 } \\
\hline
\end{tabular}


As a representative example, Figure 1 displays the calculated intermediates 7 and the energy profiles for pathways leading to the $R$ and $S$ enantiomers respectively, for the reaction promoted by catalyst $(\boldsymbol{R})-\mathbf{4 a}$.

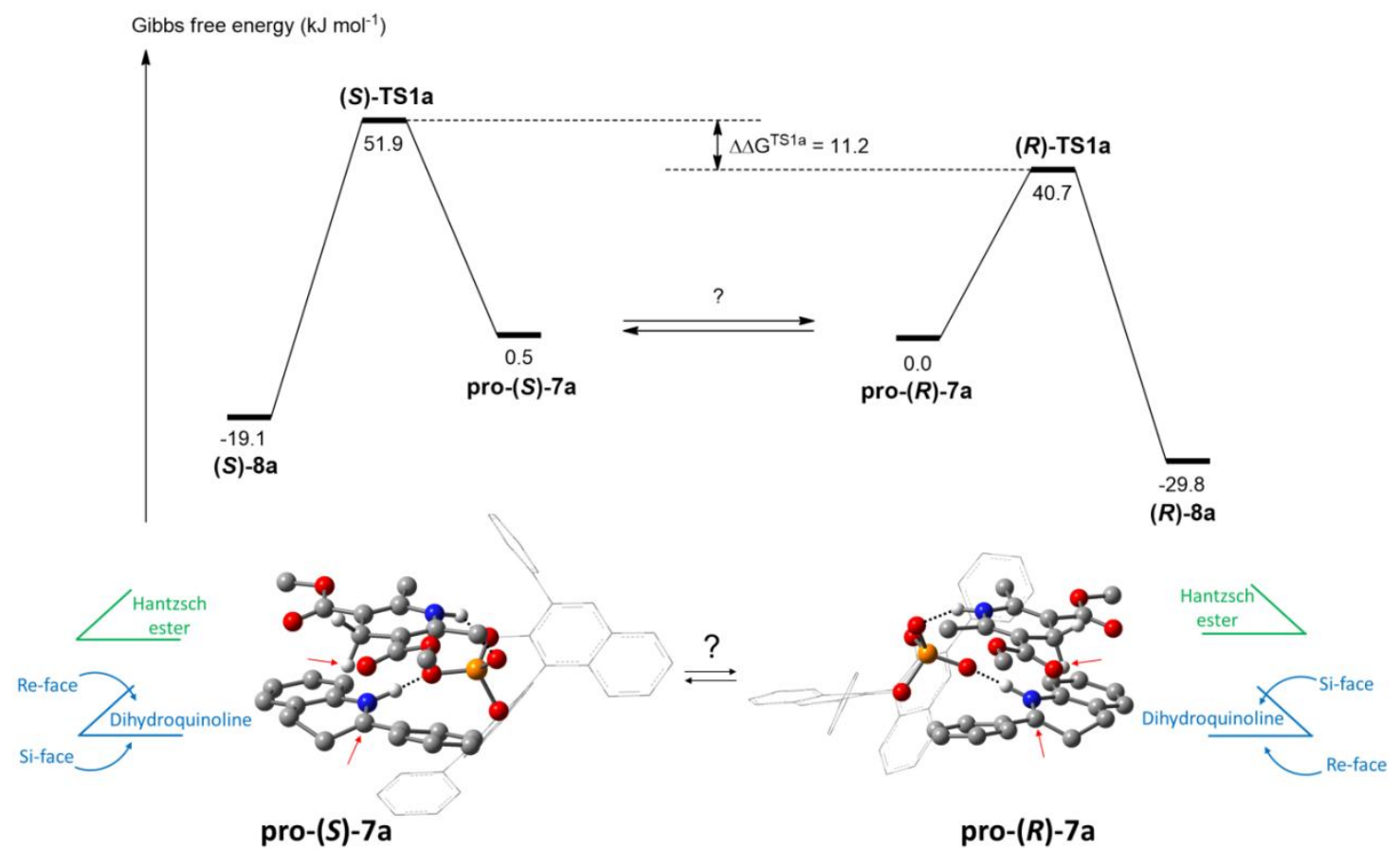

Figure 1. Top: Computed Gibbs free energies for the hydride transfer step using catalyst (R)-4a. Bottom: optimized structures of pro-(R)-7a and pro-(S)-7a. For clarity, a wireframe representation of the biaryl scaffold of $\mathbf{4 a}$ is used, and the $\mathrm{H}$ atoms have been omitted, except those involved in $\mathrm{H}$ bonding and the hydrogen atom to be transferred to the $\mathrm{C} 2$ position of DHQ (these $\mathrm{H}$ and $\mathrm{C}$ atoms are pointed by the red arrows).

The calculated free energy differences between the pro- $(R)$ and pro- $(S)$ TS1 transition states, noted as $\Delta \Delta G^{\text {TS1 }}$, for the whole series of phosphoric acids $(\mathrm{PA}=\mathbf{4 a - h})$ and the expected enantiomeric excesses are listed in Table 1. The experimental ee values are also given in Table $1 .{ }^{52}$ Our calculations showed that, in $(\boldsymbol{S})$-TS1 and $(\boldsymbol{R})$-TS1, both DHQ and HE are linked of the catalyst oxygen atoms through hydrogen bonds, irrespective of the acid used. This result is in total agreement with previous calculations. ${ }^{53,54}$ To our surprise, however, neither quantitative, nor qualitative agreement could be found between experiments and theory on several points. Contrary to the experiments, our calculations indicate that the preferred configuration of the final product may depend on the nature of the substituent in the PA catalyst: for $(\boldsymbol{R})-\mathbf{4 a}(\mathrm{Ar}=\mathrm{Ph})$ and $(\boldsymbol{R})-\mathbf{4 b}$ (Ar = 2-naphtyl), the $(R)$ enantiomer of the final tetrahydroquinoline is favored (entries 1,2$)$, 
while the $(S)$ enantiomer is favored for all other substituents. Other major inconsistencies have been noticed concerning the ee values: even for catalysts $\mathbf{4 a}$ and $\mathbf{4 h}$, which are known (4a) or expected $(\mathbf{4 h})^{55}$ to induce poor enantioselectivity for this reaction, free energy differences of 11.2 and $3.2 \mathrm{~kJ} \mathrm{~mol}^{-1}$ have been calculated respectively, which would correspond to excellent to good enantioselectivity.

Calculations with other functionals showed that the results are reproducible at different levels of theory (see Table S1 in the Supporting Information for details). Other transition states were sought for the hydride transfer step for catalyst $\mathbf{4 a}$, but all other conformations of the $(S)$ and $(R)$ transition states were found to be higher in energy than the previously computed $(\boldsymbol{S})$-TS1a and (R)-TS1a (see part II.a in the Supporting Information for details). These results finally led us to reconsider in more depth the mechanism under study.

The model used to compute the ee in Table 1, based on the free energy difference between the TS leading to each enantiomer, relies on the Curtin-Hammett principle, which requires that intermediates pro- $(\boldsymbol{R})-7$ and pro- $(\boldsymbol{S})-7$ interconvert rapidly and lead then irreversibly to $(\boldsymbol{R})-8 \mathbf{a}$ and $(\boldsymbol{S})-\mathbf{8 a}$, respectively (Figure 2). ${ }^{56}$ Examination of the structure of pro- $(\boldsymbol{R})-\mathbf{7}$ and pro- $(\boldsymbol{S})-\mathbf{7}$ in Figure 1 indicates that DHQ and HE are stacked in nearly parallel planes and that both form hydrogen bonds with the PA. Such constrained conformations, which are required to allow $\mathrm{H}$ transfer step, prevent rotation of DHQ and the subsequent easy interconversion of the pro-(R)-7 and pro- $(S)-7$ intermediates. To this end, different pathways have been considered, namely the dissociation or the deformation of pro- $(\boldsymbol{R})-7$ and pro- $(S)-7$ intermediates to allow a permutation between the $R e$-face and $S i$-face of DHQ, and we have always found a larger energetic barrier compared to the hydride transfer elementary step (see part II.b in the Supporting Information for details). This suggests that the Curtin-Hammett principle does not apply as such for the $H$ transfer step starting from the three-component complex 7.

These thoughts led us to turn our attention to reaction steps and intermediates prior to the hydride transfer step, looking for other factors which could possibly control the stereochemistry of the reaction. ${ }^{57}$ We have investigated the entire reduction cycle by using $(\boldsymbol{R})-\mathbf{4 a}$ as the catalyst (Figure 2). The first step of the catalytic cycle generates the dihydroquinolinium phosphates 6 whose conformers pro- $(\boldsymbol{R})-\mathbf{6 a}$ and pro-(S)-6a are displayed in Figure 2. It must be noted that initial coordination of HE 3 to the phosphoric acid, leading to 10a (see the Supporting Information for details), has been considered, but hasn't been retained, being clearly less exergonic. 


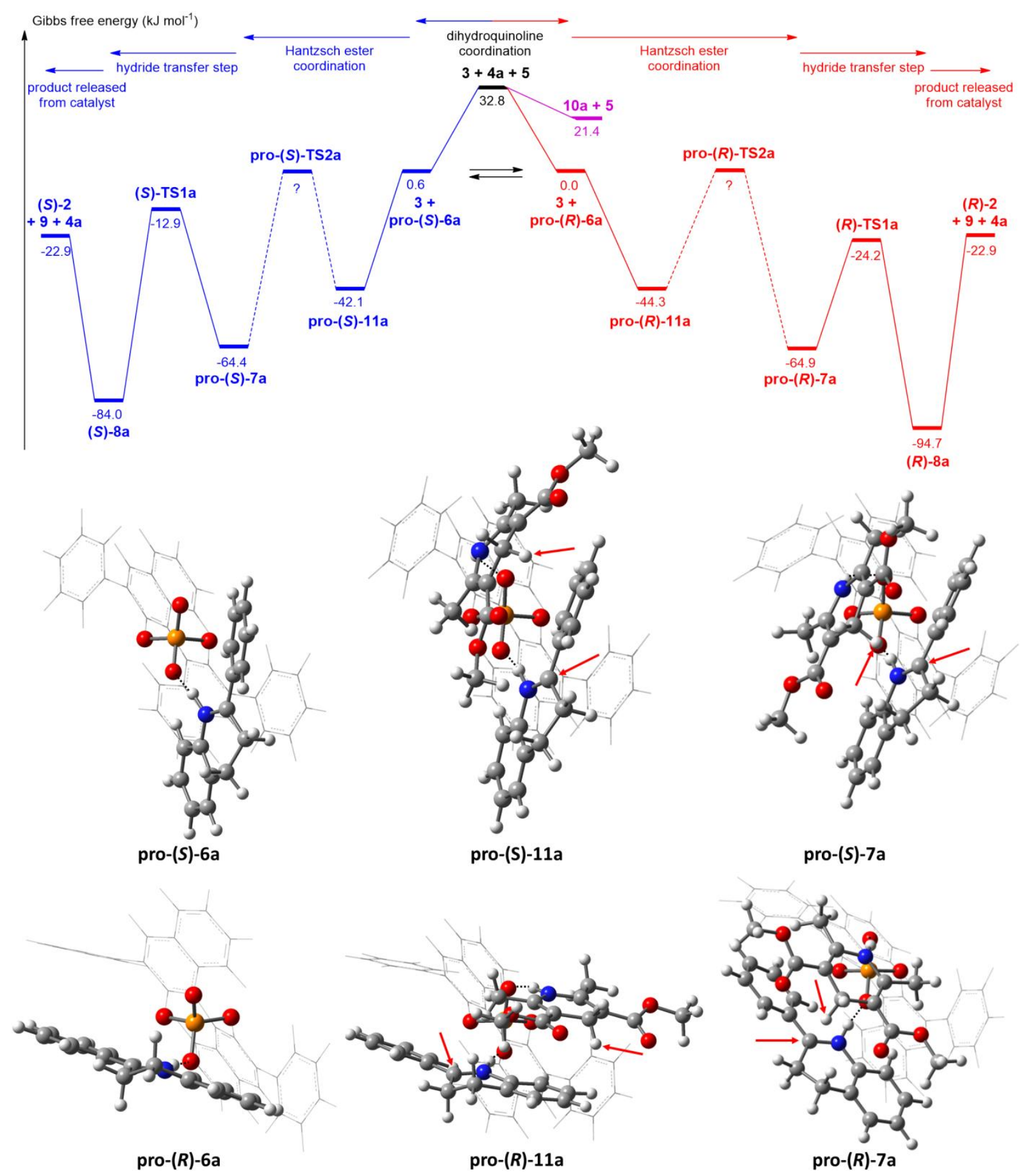

Figure 2. Computed Gibbs free energies for the reduction cycle with catalyst $(\boldsymbol{R})-\mathbf{4 a}$ and optimized structures of some intermediates. A wireframe representation of the biaryl scaffold of the PA is used for clarity. The $\mathrm{H}$ atom of the Hantzsch ester which will be transferred and the $\mathrm{C} 2$ position of DHQ are pointed by red arrows. For the easy isomerization between pro- $(\boldsymbol{R})-6 \mathbf{a}$ and pro-(S)-6a, see text and SI (part II.d). The energy level of pro-( $\boldsymbol{R})$-TS2a and pro-(S)-TS2a is arbitrary. 
In pro- $(\boldsymbol{R})-6 \mathbf{a}$ and pro-(S)-6a, DHQ presents one of its faces to the PO group on which HE 3 will coordinate (Figure 2). Interconversion between the two conformers pro- $(\boldsymbol{R})-\mathbf{6 a}$ and pro- $(\boldsymbol{S})-\mathbf{6 a}$ is expected to take place easily through rotation along the $\mathrm{PO}{ }^{\cdots} \mathrm{HN}$ axis. The activation barrier of this isomerization pathway is found at about $15 \mathrm{~kJ} \mathrm{~mol}^{-1}$, which confirms its feasibility (see part II.d in the Supporting Information for details). This easy isomerization between pro-(R)-6a and pro-(S)-6a agrees with recent study indicating that two structures are in fast exchange on the NMR time scale for imine / BINOL-derived phosphoric acids complexes by either rotating the imine by $180^{\circ}$ or switching the oxygen that forms a hydrogen bond with the imine..$^{58}$

In the next step, we have attempted optimization of the pathways leading to the three component complexes 7, by starting from pro-(R)-6a or pro-(S)-6a and by adding a HE $\mathbf{3}$ molecule from a position located outside of the catalytic pocket, but close to its entrance (see part II.e in the Supporting Information for details). Surprisingly, this pathway did not lead to the expected pro$(\boldsymbol{R})-7 \mathbf{a}$ or pro- $(S)-7 \mathbf{a}$. We obtained instead the new intermediates pro-( $\boldsymbol{R})-\mathbf{1 1 a}$ and pro-(S)-11a (Figure 2), in which both DHQ and HE form H-bond with the phosphate group of the PA, but their relative positions do not allow hydride transfer. Finding the fully optimized transition states for the association of $\mathrm{HE}$ to pro-(R/S)-6a, either connecting 11a and 7a or between $\mathbf{6 a}+\mathbf{3}$ and 7a, proves to be infeasible, probably due to the high flexibility of these three-component structures (see part II.f in the Supporting Information for details). Nevertheless, this means that, contrary to what is depicted in Scheme 2, formation of pro- $(\boldsymbol{R} / S)-7$ a from pro- $(\boldsymbol{R} / \boldsymbol{S})-\mathbf{6 a}$ requires several steps.

As shown in Figure 1, the hydride transfer step requires that DHQ and HE are properly stacked, with the $\mathrm{CH}_{2}$ group of $\mathrm{HE}$ located above the C2-carbon of DHQ. In order to obtain such a conformation from pro- $(\boldsymbol{R} / \boldsymbol{S})-\mathbf{6 a}$, it proves necessary to distort the active site, giving pro- $(\boldsymbol{R} / \boldsymbol{S})$ 12a, whose conformation corresponds exactly to that of the corresponding moiety in pro- $(\boldsymbol{R} / S)$ 7a (Figure 3). To be more precise, pro- $(\boldsymbol{R} / S)-12$ matches the frozen pro- $(\boldsymbol{R} / S)-7$ minus the HE 3 fragment. It should be noted that pro- $(\boldsymbol{R} / S)-\mathbf{1 2}$ is not a transition state, but it models the geometric deformation required to allow HE to enter into the active site. The deformation of pro$(\boldsymbol{R} / \boldsymbol{S})$-6a into pro- $(\boldsymbol{R} / \boldsymbol{S})-\mathbf{1 2 a}$ opens the gate of the active site to HE $\mathbf{3}$, and allows then formation of pro-(R/S)-7a without further deformation. 


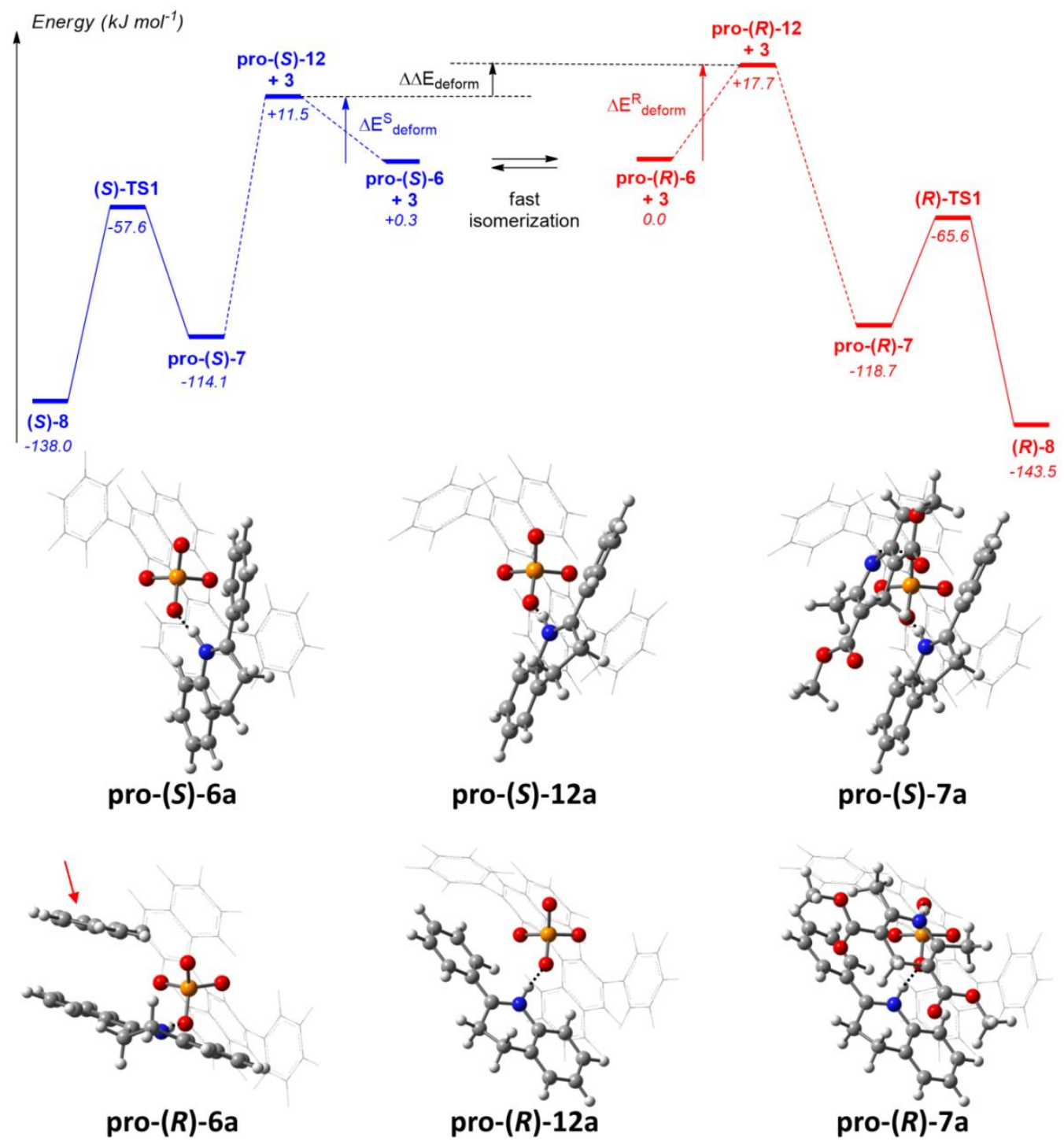

Figure 3. Model of enantioselectivity for the $H$-transfer hydrogenation of quinolines catalyzed by BINOL-derived PA and some optimized structures along these reaction pathways for PA 4a. Values indicated on the potential energy surface are electronic energy computed at the IEFPCM(toluene)-M06/6-311+G(2d,2p)//M06/6-31G(d,p) level and are given for catalyst 4a. Calculations on pro- $(\boldsymbol{R} / \boldsymbol{S})-\mathbf{1 2}$, which are not optimized geometries but binary PA-DHQ complexes exactly in their conformations computed in pro-(R/S)-7a, don't allow obtaining Gibbs free energies. Dashed lines have been used to connect pro- $(R / S)-12$ with pro- $(R / S)-6$ and pro$(\boldsymbol{R} / S)-\mathbf{7}$ because the exact reaction does not go through structure pro- $(\boldsymbol{R} / \boldsymbol{S})-\mathbf{1 2}$. A wireframe representation of the biaryl scaffold of PA is used for clarity. Only the phenyl group which inhibits insertion of HE on pro-( $(\boldsymbol{R})-\mathbf{6 a}$ is shown in 'ball-and-sticks' form and pointed by a red arrow. 
Interestingly, pro-(S)-6a and pro-(S)-12a show small differences, with only a tilt of DHQ that moves away its phenyl substituent from the PO group where HE $\mathbf{3}$ will coordinate. On the contrary, a huge variation of the geometry is observed going from pro- $(\boldsymbol{R})-6 \mathbf{a}$ to pro- $(\boldsymbol{R})-\mathbf{1 2 a}$. In pro-(R)-6a a phenyl substituent of the catalyst, which is parallel to the phenyl group of DHQ, prevents insertion of HE and, therefore, DHQ is required to move significantly away from this substituent to allow insertion (Figure 3). The calculated energy needs for the deformation of pro$(\boldsymbol{R} / \boldsymbol{S})-\mathbf{6 a}$ into pro- $(\boldsymbol{R} / \boldsymbol{S})-\mathbf{1 2} \mathrm{a}, \Delta \mathrm{E}^{\mathrm{R}}$ deform and $\Delta \mathrm{E}_{\text {deform, }}^{\mathrm{S}}$ confirm the larger geometrical change along the $(R)$-pathway, with respective values of 11.2 and $17.7 \mathrm{~kJ} \mathrm{~mol}^{-1}$.

This analysis suggests a new model to explain the enantioselectivity of this $H$-transfer reduction. The enantiodetermining step would not be the hydride transfer step $\mathbf{7} \rightarrow \mathbf{8}$ but rather the coordination of HE 3 to the PA-DHQ complex 6, i.e. step $6 \rightarrow 7$ (Figure 3). Three key points explain the good enantiocontrol of this catalytic reaction: $(i)$ a fast equilibrium is established between the pro- $(\boldsymbol{R})$ and pro- $(\boldsymbol{S})$ conformers of the PA-DHQ adducts 6; (ii) the lower energy pathway allows insertion of HE into one of the PA-DHQ adduct 6 to afford selectively pro-(S)-7;

(iii) HE insertion to form the three-component complexes 7 is highly exergonic and irreversible, as hydride transfer is the easiest way from 7. This catalytic reaction is therefore under CurtinHammett control and the origin of enantiocontrol relates to "dynamic kinetic selection" between two prochiral conformers.

Calculation of the barrier height for step $\mathbf{6} \rightarrow \mathbf{7}$ could assess the validity of this enantiocontrol model. Despite numerous attempts (see part II.f in the Supporting Information for details), transition states for step $\mathbf{6} \rightarrow \mathbf{7}$ could not be identified, therefore the $\mathbf{6} \rightarrow \mathbf{7}$ barrier height could not be calculated directly. We therefore turn our computations to an approximate approach. The exact pathway from 6 to 7 implies simultaneously both structural deformation of the PA-DHQ complex 6 and insertion of HE 3 into this complex (i.e. active site). These two steps have been considered successives. The first one, leading to 12, is endothermic (deformation of the geometry-optimized 6 complex), whereas the second one is exothermic (formation of the H-bond between PA and HE without deformation). It is expected that this split-up description of the $\mathbf{6} \rightarrow$ 7 multimolecular reaction could bring insight into such chemical step for which it has been indicated previously that the ability of calculations could be questionable. ${ }^{44}$ Indeed, the change in energy from pro- $(\boldsymbol{R} / \boldsymbol{S})-\mathbf{6}$ to pro- $(\boldsymbol{R} / \boldsymbol{S})-\mathbf{1 2}$ corresponds to the deformation energy of the PA-DHQ 
fragment (i.e. the active site) when going from $3+\operatorname{pro}-(\boldsymbol{R} / S)-6$ to pro- $(\boldsymbol{R} / S)-7$. Therefore, the energy increase from $\mathbf{6}$ to $\mathbf{1 2}$ is anticipated to parallel well the barrier height for step $\mathbf{6} \rightarrow \mathbf{7}$ and thus could be used to compute the enantiomeric excess.

Using this model, the catalyst control of the enantiomeric ratio can be quantified by computing the energy difference $\left(\Delta \Delta \mathrm{E}_{\text {deform}}\right)$ between pro- $(\boldsymbol{R})-12$ and pro- $(S)-12$ (Figure 3). $\Delta \Delta \mathrm{E}_{\text {deform }}$ were computed for all catalysts $(\boldsymbol{R})-\mathbf{4 a - f}$ that have been screened experimentally. In all cases, pro-( $\boldsymbol{R})$ 12 is found to be higher in energy than pro-(S)-12, in agreement with the experiments which indicate that the $(S)$ enantiomer is favored for all catalysts. Furthermore, the new model above allows quantitative agreement between experiment and theory. Indeed, an excellent correlation between the experimental enantiomeric excesses and the computed $\Delta \Delta \mathrm{E}_{\text {deform }}$ is obtained for the whole set of catalysts $\left(\mathrm{R}^{2}=0.96\right.$, Figure 4 and Table 2$)$.

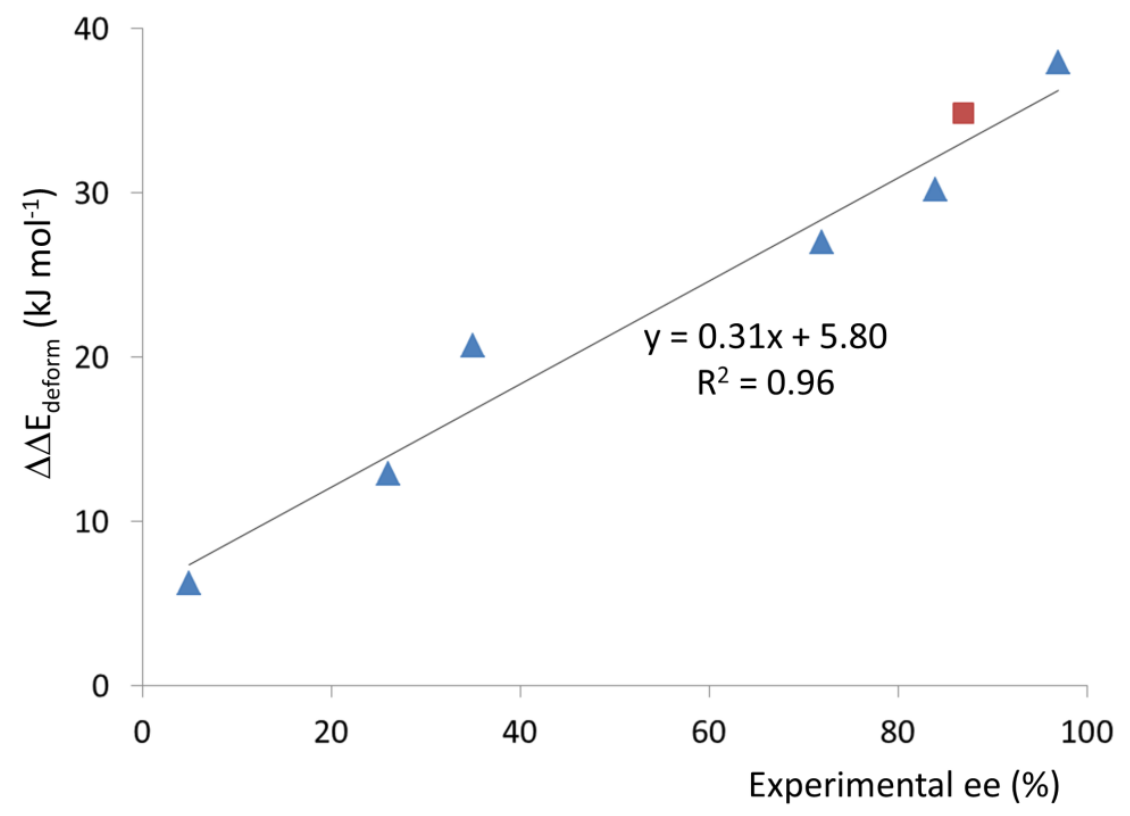

Figure 4. Plot of the computed energy difference between pro- $(\boldsymbol{R})-\mathbf{1 2}$ and pro- $(S)-12\left(\Delta \Delta \mathrm{E}_{\text {deform }}\right)$ vs. experimental ee in the reduction of 2-phenyl-quinoline with catalysts $(\boldsymbol{R})-\mathbf{4 a - f}$ (blue triangles) and reduction of $2-{ }^{n} \mathrm{Bu}$-quinoline with $(\boldsymbol{R})-\mathbf{4 f}$ (red square). The correlation line is based only on the values obtained for the reduction of 2-phenyl-quinoline with $(\boldsymbol{R})-\mathbf{4 a - f}$. 
Table 2. DFT-predicted vs. experimental ee in the reduction of 2-substituted-quinoline with catalysts $(\boldsymbol{R})-\mathbf{4 a - f}$.

\begin{tabular}{|c|c|c|c|c|c|}
\hline \multirow{2}{*}{ entry } & \multirow[t]{2}{*}{ Cat. } & \multirow{2}{*}{$\begin{array}{c}\text { 2-R-quinoline } \\
\mathrm{R}\end{array}$} & \multirow{2}{*}{$\begin{array}{l}\Delta \Delta \mathrm{E}_{\text {deform }} \\
\left(\mathrm{kJ} \mathrm{mol}^{-1}\right)\end{array}$} & \multirow{2}{*}{$\begin{array}{l}\text { Predict. } \\
\text { ee }[\%]^{\mathrm{a}}\end{array}$} & \multirow{2}{*}{$\begin{array}{l}\text { Exper. } \\
e e[\%]^{\mathrm{b}}\end{array}$} \\
\hline & & & & & \\
\hline 1 & $4 a$ & $\mathrm{Ph}$ & 6.2 & 1 & 5 \\
\hline 2 & $4 b$ & $\mathrm{Ph}$ & 12.9 & 23 & 26 \\
\hline 3 & $4 c$ & $\mathrm{Ph}$ & 20.7 & 48 & 35 \\
\hline 4 & $4 d$ & $\mathrm{Ph}$ & 27.0 & 68 & 72 \\
\hline 5 & $4 e$ & $\mathrm{Ph}$ & 30.2 & 79 & 84 \\
\hline 6 & $4 f$ & $\mathrm{Ph}$ & 37.9 & 100 & 97 \\
\hline 7 & 4f & ${ }^{n} \mathrm{Bu}^{\mathrm{c}}$ & 34.8 & 94 & 87 \\
\hline
\end{tabular}

${ }^{a}$ Predicted ee values computed based on the slope and the intercept of the correlation line shown in Figure 4: ee $=$ $\left(\Delta \Delta \mathrm{E}_{\text {deform }}-5.80\right) / 0.31$. All calculations predict $(S)$-configured tetrahydroquinoline products. ${ }^{\mathrm{b}}$ Experimental values from ref. 52. All experiments afford $(S)$-configured tetrahydroquinoline products. ${ }^{\mathrm{c}}$ Calculations performed for the 2methyl-quinoline.

In order to test the robustness of our model, we have applied it also to the reduction of 2- $n$-butylquinoline catalyzed by $(\boldsymbol{R})-\mathbf{4 f} .^{52}$ Calculations, which have been performed with the simpler 2 methyl-quinoline model, have predicted that the $(S)$ enantiomer of the final tetrahydroquinoline is favored with an enantiomeric excess of $94 \%$, in good agreement with the experimental value (87 $\%$, Figure 4 and Table 2).

Thus, these calculations accurately reproduce all aspects of the stereochemical outcome of $H$ transfer reactions on two different substrates, within an homogeneous series of acid catalysts and a good correlation is obtained between experimental and predicted ee. They make it possible to provide a new proposal for the enantiocontrol in phosphoric acid catalyzed $H$-transfer hydrogenation of quinoline. A simplified representation of the mechanism is given in Figure 5 and compared to the previously accepted scheme. The enantiodetermining step is not the hydride transfer step but rather the association of the Hantzsch ester to the catalyst-substrate complex. Moreover, the calculations also allow some understanding of the origin of enantiocontrol from the chiral catalysts, as a function of the aryl substituents on their biaryl scaffolds. Indeed, in all cases computed so far, pro- $(\boldsymbol{R})-6$ is always slightly lower in free energy than pro- $(S)-6(\Delta=0.6$ $8.8 \mathrm{~kJ} \mathrm{~mol}^{-1}$ ) and the deformation energy of pro-(S)-6 to form pro-(S)-12 is uniformly small (see 
the Supporting Information for details). On the contrary, the deformation energy of pro-(R)-6 to form pro-( $\boldsymbol{R})-12$ varies significantly depending on the nature of the catalyst, ranging from 17.7 to $42.7 \mathrm{~kJ} \mathrm{~mol}^{-1}$. This large deformation energy of the active site required to allow the Hantzsch ester insertion can be associated to the presence of one aryl substituent of the catalyst close to the free $\mathrm{P}=\mathrm{O}$ group in pro-(R)-6 (Figure 3). Two effects may account for the influence of this substituent on the deformation energy: $(i)$ in pro- $(\boldsymbol{R})-\mathbf{6}$, the observed intermolecular interactions between DHQ and one of the aryl substituents of PA might depend strongly on the nature of the aryl ring; (ii) adjustment of the active site to allow entrance of HE might also be affected by the size and geometry of these aryl substituents.

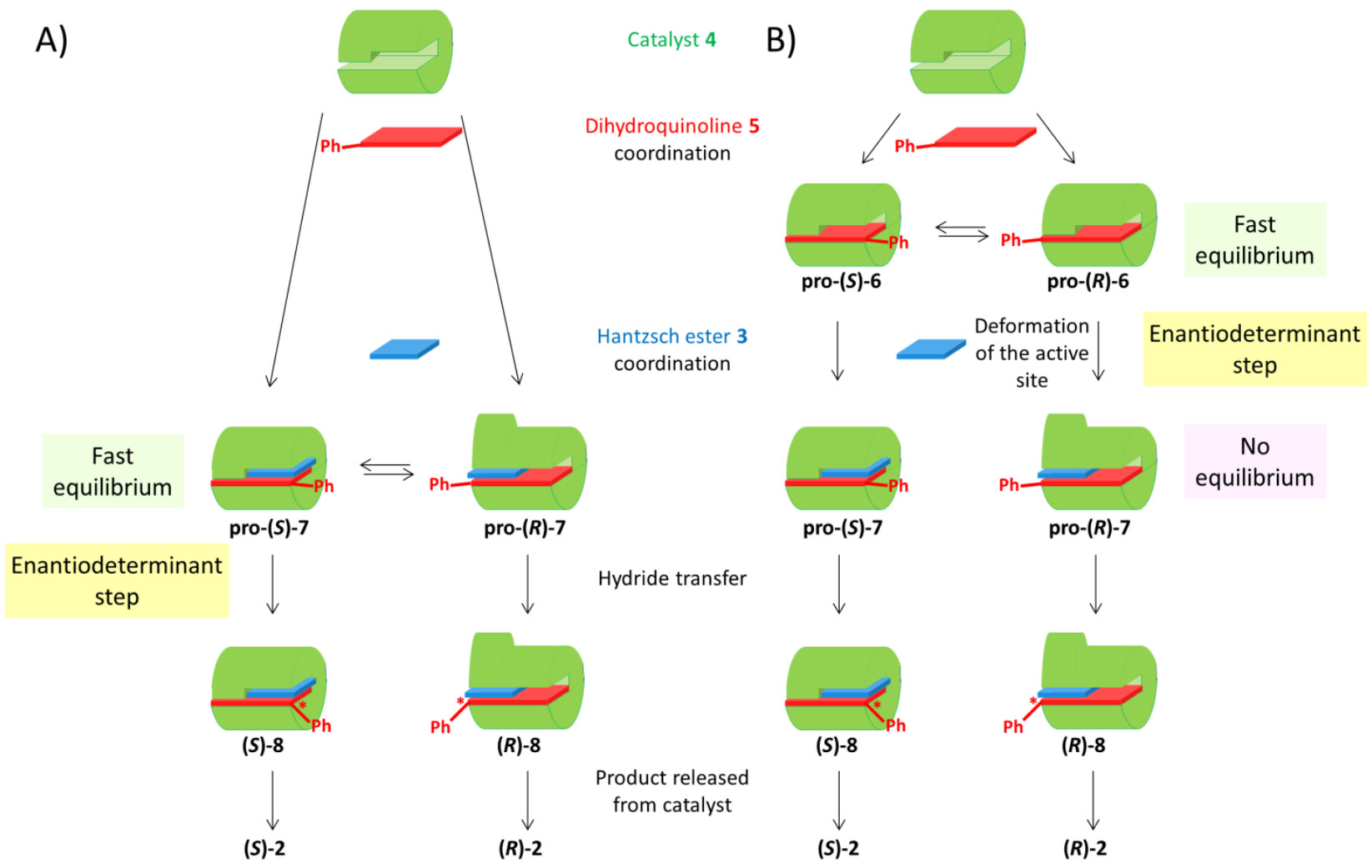

Figure 5. Previous (A) and proposed (B) mechanisms for the enantiocontrol in phosphoric acid catalyzed $H$-transfer hydrogenation of quinolone

\section{Conclusion}

In conclusion, we have proven through state-of-the-art calculations that the previously accepted mechanistic proposals for the stereochemical control in the organocatalytic $H$-transfer hydrogenation of quinolones are inadequate to explain the enantiomeric excesses of the reaction. 
The simple modeling of the transition state of hydride transfer, elemental step of the catalytic cycle where the stereogenic center is generated, is not sufficient to fully account of enantioselectivity. A thorough re-examination of the mechanistic steps allows to put forward an alternative proposal in which the enantioselectivity would be achieved by selective association. According to this model, using a $(R)$-BINOL-derived PA, stereochemical control occurs through the preferential formation of the three-component complex pro- $(S)-7$, with respect to pro- $(\boldsymbol{R})-\mathbf{7}$, since HE insertion in the pro- $(R)$ active site is far more difficult. Calculations based on this model accurately reproduce experimental data for the first time, and provide to enlighten about the origin of the enantioselectivity. Indeed, one aryl substituent of the catalyst has been identified to inhibit insertion of HE only for the $(R)$-path. This explains the larger deformation of the active site required for the $(R)$-path compared to the $(S)$-path. Ongoing research in our group aims to establish the applicability of this model to other organocatalytic processes.

\section{Supporting information}

Computational methods; Numerous computational details; Cartesian coordinates and absolute electronic energies of stationary points.

\section{Conflicts of interest}

The authors declare no conflict of interest.

\section{Acknowledgements}

We are grateful to the Agence Nationale de la Recherche (ANR) for financial support to this work, within the ANR Blanc "Chiracid" project. This work was performed using HPC resources from GENCI-CINES/IDRIS (Grant 2016-086894). Region Ile-de-France is acknowledged for a PhD scholarship to J.P.

\section{References}

(1) Sheldon, R. A. Chem. Soc. Rev. 2012, 41, 1437-1451.

(2) Green Catalysis: Homogeneous Catalysis in Handbook of Green Chemistry; Anastas, P. T., Crabtree, R. H., Eds.; Wiley-VCH, 2013; Vol. 1, 431 pages.

(3) Scheffler, U.; Mahrwald, R. Chem. Eur. J. 2013, 19, 14346-14396. 
(4) Chirik, P. J.; Wieghardt, K. Science 2010, 327, 794-795.

(5) Luca, O. R.; Crabtree, R. H. Chem. Soc. Rev. 2013, 42, 1440-1459.

(6) Bauer, I.; Knoelker, H.-J. Chem. Rev. 2015, 115, 3170-3387.

(7) Zhou, J. Chem. Asian J. 2010, 5, 422-434.

(8) Lohr, T. L.; Marks, T. J. Nat. Chem. 2015, 7, 477-482.

(9) Sameera, W. M. C.; Maseras, F. WIREs Comput. Mol. Sci. 2012, 2, 375-385.

(10) Cheong, P. H.-Y.; Legault, C. Y.; Um, J. M.; Celebi-Ölçüm, N.; Houk, K. N. Chem. Rev. 2011, 111, 5042-5137.

(11) Sperger, T.; Sanhueza, I. A.; Kalvet, I.; Schoenebeck, F. Chem. Rev. 2015, 115, 95329586.

(12) Cheng, G. J.; Zhang, X.; Chung, L. W.; Xu, L.; Wu, Y. D. J. Am. Chem. Soc. 2015, 137, 1706-1725.

(13) Lam, Y.; Grayson, M. N.; Holland, M. C.; Simon, A.; Houk, K. N. Acc. Chem. Res. 2016, $49,750-762$.

(14) Santoro, S.; Kalek, M.; Huang, G.; Himo, F. Acc. Chem. Res. 2016, 49, 1006-1018.

(15) Sperger, T.; Sanhueza, I. A.; Schoenebeck, F. Acc. Chem. Res. 2016, 49, 1311-1319.

(16) Kiss, G.; Celebi-Ölcüm, N.; Moretti, R.; Baker, D.; Houk, K. N. Angew. Chem. Int. Ed. 2013, 52, 5700-5725.

(17) Świderek, K.; Tuñón, I.; Moliner, V. WIREs Comput. Mol. Sci. 2014, 4, 407-421.

(18) Robles, V. M.; Ortega-Carrasco, E.; Alonso-Cotchico, L.; Rodriguez-Guerra, J.; Lledos, A.; Maréchal, J.-D. ACS Catal. 2015, 5, 2469-2480.

(19) Nørskov, J. K.; Bligaard, T.; Rossmeis, J.; Christensen, C. H. Nat. Chem. 2009, 1, 37-46.

(20) Houk, K. N.; Cheong, P. H.-Y. Nature 2008, 455, 309-313.

(21) Jover, J.; Fey, N. Chem. Asian J. 2014, 9, 1714-1723.

(22) Reid, J. P.; Simon, L.; Goodman, J. M. Acc. Chem. Res. 2016, 49, 1029-1041.

(23) Chu, Y. H.; Heyndrickx, W; Occhipinti, G.; Jensen, V. R.; Alsberg, B. K. J. Am. Chem. Soc. 2012, 134, 8885-8895.

(24) Houk, K. N.; Liu, F. Acc. Chem. Res. 2017, 50, 539-543.

(25) Hammes-Schiffer, S. Acc. Chem. Res. 2017, 50, 561-566.

(26) Poree, C.; Schoenebeck, F. Acc. Chem. Res. 2017, 50, 605-608. 
(27) Harriman, D. J.; Lambropoulos, A.; Deslongchamps, G. Tetrahedron Lett. 2007, 48, 689692.

(28) Kozlowski, M. C.; Dixon, S. L.; Panda, M.; Lauri, G. J. Am. Chem. Soc. 2003, 125, 66146615.

(29) Ianni, J. C.; Annamalai, V.; Phuan, P.-W.; Panda, M.; Kozlowski, M. C. Angew. Chem. Int. Ed. 2006, 46, 5502-5505.

(30) Harper, K. C.; Bess, E. N.; Sigman, M. S. Nat. Chem. 2012, 4, 366-374.

(31) Harper, K. C.; Sigman, M. S. J. Org. Chem. 2013, 78, 2813-2818.

(32) Milo, A.; Neel, A. J.; Toste, F. D.; Sigman, M. S. Science 2015, 347, 737-743.

(33) Sigman, M. S.; Harper, K. C.; Bess, E. N.; Milo, A. Acc. Chem. Res. 2016, 49, 12921301.

(34) (a) Reid, J. P.; Goodman, J. M. J. Am. Chem. Soc. 2016, 138, 7910-7917; (b) Reid, J. P.; Goodman, J. M. Chem. Eur. J. 2017, 23, 14248-14260.

(35) Mori, K.; Ichikawa, Y.; Kobayashi, M.; Shibata, Y.; Yamanaka, M.; Akiyama, T. Chem. Sci. 2013, 4, 4235-4239.

(36) Balcells, D.; Maseras, F. New J. Chem. 2007, 31, 333-343.

(37) Hansen, E.; Rosales, A. R.; Tutkowski, B.; Norrby, P.-O.; Wiest, O. Acc. Chem. Res. 2016, 49, 996-1005.

(38) Sunoj, R. B. Acc. Chem. Res. 2016, 49, 1019-1028.

(39) Donoghue, P. J.; Helquist, P.; Norrby, P.-O.; Wiest, O. J. Am. Chem. Soc. 2009, 131, 410411.

(40) Bahmanyar, S.; Houk, K. N.; Martin, H. J.; List, B. J. Am. Chem. Soc. 2003, 125, 24752479.

(41) Akiyama, T.; Morita, H.; Bachu, P.; Mori, K.; Yamanaka, M.; Hirata, T. Tetrahedron 2009, 65, 4950-4956.

(42) Lu, T.; Zhu, R.; An, Y.; Wheeler, S. E. J. Am. Chem. Soc. 2012, 134, 3095-3102.

(43) Rooks, B. J.; Haas, M. R.; Sepúlveda, D.; Lu, T.; Wheeler, S. E. ACS Catal. 2015, 5, 272280.

(44) Plata, R. E.; Singleton, D. A. J. Am. Chem. Soc. 2015, 137, 3811-3826.

(45) Hopmann, K. H. Int. J. Quantum Chem. 2015, 115, 1232-1249.

(46) Rohmann, K.; Hölscher, M.; Leitner, W. J. Am. Chem. Soc. 2016, 138, 433-443. 
(47) Terada, M. Synthesis 2010, 1929-1982.

(48) Parmar, D.; Sugiono, E.; Raja, S.; Rueping, M. Chem. Rev. 2014, 114, 9047-9153.

(49) Akiyama, T.; Itoh, J.; Yokota, K.; Fuchibe, K. Angew. Chem. Int. Ed. 2004, 43, 15661568.

(50) Uraguchi, D.; Terada, M. J. Am. Chem. Soc. 2004, 126, 5356-5357.

(51) Faisca Phillips, A. M.; Pombeiro, A. J. L. Org. Biomol. Chem. 2017, 15, 2307-2340.

(52) Rueping, M.; Antonchick, A. P.; Theissmann, T. Angew. Chem. Int. Ed. 2006, 45, 36833686.

(53) Simón, L.; Goodman, J. M. J. Am. Chem. Soc. 2008, 130, 8741-8747.

(54) Marcelli, T.; Hammar, P.; Himo, F. Chem. Eur. J. 2008, 14, 8562-8571.

(55) More, G. V.; Bhanage, B. M. Tetrahedron-Asymmetry 2015, 26, 1174-1179.

(56) Hopmann, K. H.; Frediani, L.; Bayer, A. Organometallics 2014, 33, 2790-2797.

(57) Bures, J.; Armstrong, A.; Blackmond, D. G. Acc. Chem. Res. 2016, 49, 214-222.

(58) Greindl, J.; Hioe, J.; Sorgenfrei, N.; Morana, F.; Gschwind, R. M. J. Am. Chem. Soc. 2016, 138, 15965-15971. 


\section{Table of contents entry}
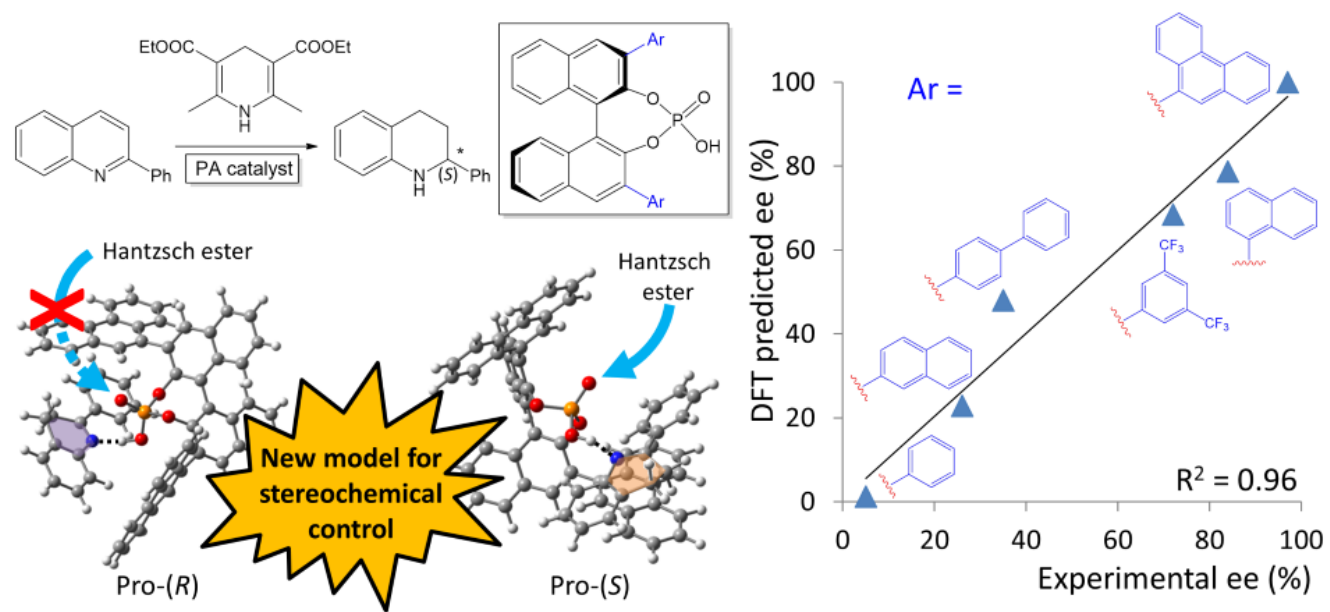